\title{
El cambio climático: un caso de securitización exitosa del medio ambiente María Francisca Casado Claro*
}

\section{Resumen}

A comienzos de la década de 1980, la seguridad medioambiental no formaba parte de las corrientes prevalentes en los estudios internacionales de seguridad; un mainstream del que sería sin embargo complicado imaginar que no formara parte en la actualidad. Aunque continúa desarrollándose y avanzando en la agenda política, la seguridad medioambiental ha pasado de ser inicialmente un campo controvertido y debatido a ser considerado un agravante de conflictos o condiciones preexistentes y de ahí a tener entidad propia, impulsado por preocupaciones existenciales de primer orden, como el cambio climático en la época del Antropoceno.

Comenzamos este artículo revisando el concepto de seguridad medioambiental, para luego abordar el marco de análisis de la Escuela de Copenhague y su aplicación al sector de la seguridad medioambiental, con el objetivo de analizar la securitización exitosa del cambio climático por parte de la comunidad internacional.

\section{Palabras Clave}

Seguridad medioambiental; Estudios Críticos de Seguridad; Escuela de Copenhague; Securitización; Cambio climático.

\section{TITLE}

Climate change: a case of successful environmental securitization

\section{Abstract}

In the early 1980s, environmental security was not part of the international security studies mainstream, where it is nowadays firmly grounded. Both from the point of view of practice and theory, environmental security continues to be developed. From being considered a source of controversy or an aggravating factor of pre-existing conflicts or conditions, environmental security is today a first-order subject where existential concerns, such as climate change at the time of the Anthropocene, are addressed.

This article begins with a revision of the concept of environmental security. With the aim of analyzing the successful securitization of climate change by the international community, it looks at the analytical framework of the Copenhagen School and its application to the environmental security sector.

\section{KEYWORDS}

Environmental Security; Critical Security Studies; Copenhagen School; Securitization; Climate change.

* María Francisca CASADO CLARO, Doctora en Paz y Seguridad Internacional por la UNED, Magister en Relaciones Internacionales por la Universidad Complutense de Madrid y ha cursado estudios en la Universidad de Tokio y en la Universidad de las Naciones Unidas gracias a una beca concedida por el Ministerio de Educación (MEXT) japonés. Enseña en la Facultad de Ciencias Sociales de la Universidad Europea de Madrid. Su correo electrónico es: francisca. casado@universidadeuropea.es

La autora agradece a los evaluadores anónimos las sugerencias y observaciones y a la directora de la revista Marina Díaz Sanz su apoyo a lo largo del proceso de revisión.

\section{Recibido:}

2016-11-16 Aceptado: 2017-01-12 DOI: $10.15366 /$ relacionesinternaci onales2017.34.002 


\section{ntroducción}

Mientras que, por separado, la seguridad y el medio ambiente forman parte la agenda internacional desde hace mucho tiempo, la "seguridad medioambiental" tan sólo figura en la misma desde principios de los años noventa, impulsada por el final de la Guerra Fría. Con el fin del orden bipolar surgió la necesidad de redefinir el concepto de seguridad de forma que contemplase otras dimensiones de la misma. De esta manera, el concepto estatocéntrico tradicional de la seguridad como "defensa militar" se sometió a una doble dinámica de ampliación y profundización dando paso a una definición más aglutinadora ${ }^{1}$.

A través de la ampliación la seguridad se convirtió en "integral" (comprehensive security) abarcando no sólo el ámbito militar, sino también cuestiones políticas, económicas, sociales y medioambientales. Por otra parte, a través de la profundización entraron en escena nuevos objetos referentes y nuevos actores ${ }^{2}$. La inclusión de cuestiones de seguridad blanda en la agenda de seguridad trasciende la visión tradicional de los estados de su seguridad nacional, en la que es el propio estado el que se ve amenazado por acciones provenientes de más allá de sus fronteras, pues lo que se ve amenazado no es una abstracción como el estado sino el bienestar de los individuos y su forma de vida. La redefinición del concepto de seguridad desde el punto de vista teórico encendió un debate académico, que también ha encontrado su paralelo dentro de las estrategias de seguridad nacional de algunos países, como Estados Unidos ${ }^{3}$, Australia ${ }^{4}$, Japón ${ }^{5}$ o Francia ${ }^{6}$.

En este artículo revisamos la conceptualización, evolución y definición de seguridad medioambiental antes de adentrarnos en la aplicación práctica del marco de análisis de la Escuela de Copenhague al sector medioambiental, tomando como estudio de caso la securitización exitosa del cambio climático. El marco de análisis de la Teoría de la Securitización es uno de los acercamientos más pragmáticos al tratamiento de los riesgos medioambientales, lejos de ser un hito en el tiempo, el modelo sigue evolucionando y aquí comprobamos cómo se ajusta al sector de la seguridad medioambiental, a través del análisis del proceso que ha situado el cambio climático al frente de las preocupaciones de la comunidad internacional.

\section{La dificultad de acotar el término seguridad medioambiental}

Al finalizar la Guerra Fría, el desacuerdo entre los analistas de la seguridad sobre si el medio ambiente debía analizarse en términos de seguridad corría paralelo al debate en torno a la reconceptualización de la seguridad. Mientras para unos encuadrar los problemas

\footnotetext{
1 Véase BROWN, Lester Russell, Redefining national security, vol. 14, Worldwatch Institute, Washington D.C., 1977; ULLMAN, Richard H., "Redefining security", en International Security, no 8, 1983; BALDWIN, David A. "The concept of security", en Review of International Studies, 23.01, 1997, ps. 5-26; SMITH, Steve, "The contested concept of security", en BOOTH, Ken (ed.), Critical Security Studies and World Politics, Lynne Rienner Publishers, Boulder, Colorado, 2005.

2 Véase BUZAN, Barry, WÆVER, Ole y DE WILDE, Jaap, Security: a new framework for analysis, Lynne Rienner Publishers, Londres, 1998; COLLINS, Alan (ed), Contemporary Security Studies, Oxford University Press, 2007.

3 FLOYD, Rita. Security and the Environment: Securitisation theory and US environmental security policy. Cambridge University Press, 2010.

4 Department of DEFENCE, AUSTRALiAn GovernmenT. Defence White Paper 2016. ps.41, 55, 56, 102.

5 National Security Strategy of Japan, en http://japan.kantei.go.jp/96_abe/documents/2013/_icsFiles/ afieldfile/2013/12/17/NSS.pdf, ps.8-9, ps. 32-34.

6 French White Paper on Defence and National Security 2013. p.40, en http://www.defense.gouv.fr/english/ content/download/206186/2393586/file/White\%20paper\%20on\%20defense\%20\%202013.pdf
} 
medioambientales desde el enfoque de la seguridad supondría militarizar el medioambiente y la "lente de la seguridad" no resulta útil para analizar el medio ambiente", para otros ese es el lugar que deben ocupar a fin de hacer frente a amenazas existenciales ${ }^{8}$.

Un punto de partida para analizar si el medio ambiente era o podía llegar a ser una cuestión de seguridad era preguntar: "¿Puede constituir el medio ambiente una amenaza a la seguridad?" La respuesta a esta pregunta podría ser afirmativa o negativa.

Desde un punto de vista realista ortodoxo, la respuesta es "no", ya que la única amenaza posible a la seguridad nacional sería un ataque armado inminente. En su opinión definir el medio ambiente en términos de seguridad despojaría de coherencia intelectual a la Seguridad, lo cual dificultaría el poder idear soluciones para problemas de seguridad graves. En este sentido los problemas medioambientales son sólo eso, "problemas", y por lo tanto no deben ser tratados en el ámbito de la seguridad por muy graves que sean. Asimismo, otros autores consideran que es analíticamente engañoso pensar en la degradación medioambiental como una amenaza a la seguridad nacional ya que el enfoque tradicional de la seguridad nacional tiene poco en común con cualquiera de los problemas medioambientales o sus soluciones. Según Deudney esto crea confusión sobre cuáles son las verdaderas tareas en las que hay que centrar la atención? .

Deudney es uno de los autores que más enérgicamente ha abogado por mantener el ambientalismo y la seguridad en compartimentos separados: "la seguridad-nacional-sinviolencia y la habitabilidad del medio ambiente son mucho más diferentes que similares", por lo que dadas las diferencias de presupuestos, "vincularlas mediante la redefinición podría crear una embrollo conceptual más que un cambio de paradigma"10. Deudney defiende que la degradación del medio ambiente no es una amenaza para la seguridad nacional, sino más bien que el ecologismo es en sí mismo una amenaza a la hegemonía de los discursos e instituciones de la seguridad nacional estatocéntrica ${ }^{11}$.

En contraposición, dentro de los Estudios Críticos de Seguridad (en adelante, ECS), la respuesta a la pregunta de si el medio ambiente puede constituir una amenaza a la seguridad, sería "sí", aunque matizada con un "pero solo bajo ciertas circunstancias". Así, para un constructivista, la pregunta que deberíamos plantearnos no es si las cuestiones medioambientales pueden "ser" o "llegar a ser" amenazas a la seguridad, sino más bien si pueden "ser consideradas" amenazas a la seguridad, ya que en la medida en que la realidad es una construcción social uno debe preguntarse no sobre la esencia o la existencia real de las amenazas que haya en el mundo, sino sobre la percepción de las mismas. La seguridad aborda fundamentalmente el estudio de la construcción social de las amenazas y el analista

\footnotetext{
7 HOUGH, Peter, Environmental Security: An introduction, Routledge, Londres, 2014, p. 139.

8 TROMBETTA, Maria Julia, "Rethinking the securitization of the environment: Old beliefs, new insights", en BALZACQ, Thierry (ed.), Securitization Theory: How security problems emerge and dissolve, Routledge, Londres, 2011 , p. 135.

9 DEUDNEY, Daniel H., "Environmental security: A critique" en DEUDNEY, Daniel H. y MATTHEW, Richard A. (eds.), Contested Grounds: Security and Conflict in the New Environmental Politics, SUNY Press, Albany, Nueva York, 1999, p. 194.

${ }_{10}$ Ibídem, p. 194.

${ }^{11}$ Ibíd., p. 214
} 
determina qué, para quién y cuándo un problema de seguridad se convierte en amenaza.

ECS es un apelativo que tiene su origen en una conferencia celebrada en la Universidad de York (Toronto, Canadá) en 1994, en la que intervinieron expertos de diferentes orientaciones ideológicas que publicaron sus artículos en un libro coordinado por Krause y Williams que vio la luz en 1997 bajo el título que más tarde daría nombre al movimiento (Critical Security Studies: Concepts and cases). Los ECS son un enfoque surgido en contraposición a la visión tradicional de la seguridad, sus defensores se plantearon si el Estado debía ser el único objeto referente (¿quién o qué debía asegurarse?), cuáles debían ser el alcance de la seguridad y de los estudios de seguridad (¿debía ampliarse el campo?) y su naturaleza (al igual que las comunidades humanas, ¿el Estado no se rige también por ideas, normas y valores?).

Mutimer argumenta que, bajo el paraguas de los ECS, Krause y Williams pretendían fomentar una "perspectiva crítica" que no fuese monopolizada por un solo enfoque teórico ${ }^{12}$. Sin embargo, a pesar de sus buenas intenciones, crearon las condiciones propicias para que se produjese un "cisma" que cambiaría el panorama de los estudios estratégicos y de seguridad. Según este autor varias escuelas intervinieron en la ruptura, aportando sus perspectivas al debate sobre el concepto de seguridad: la Escuela de Copenhague ${ }^{13}$, la Escuela de Gales ${ }^{14}$, el constructivismo y el post-estructuralismo ${ }^{15}$. Aunque Mutimer no la menciona y en general es menos conocida que las anteriores, entre otras cosas por escribir en francés mientras el debate académico tiene lugar en inglés, también ha cobrado relevancia por sus aportaciones la Escuela de París ${ }^{16}$.

Dentro de los ECS, existe un pronunciado grupismo. Buzan, Wæver y de Wilde se distancian de los ECS argumentando que estos se basan en el constructivismo y el postestructuralismo, corrientes abiertas a la posibilidad de cambio social. Copenhague reconoce la construcción social de la realidad pero la considera suficientemente estable a largo plazo, por lo que puede tratarse de forma objetiva ${ }^{17}$. Por su parte, Ken Booth manifiesta que hay momentos en los que hay que trazar una línea roja y que el espíritu que subyace en su marco de análisis es muy diferente del de Krause y Williams al invocar "una nueva ortodoxia", sin

12 El volumen conjuga enfoques que van desde el realismo de Mohammed Ayoob al constructivismo que en ese tiempo estaba influyendo sobre el debate en el campo de las Relaciones Internacionales, yendo hasta la filosofía post-estructuralista o al post-Marxismo basado en la Escuela de Frankfurt, defendido por Peter Vale y Ken Booth.

${ }^{13}$ Se originó en el COPRI (Copenhaguen Peace Research Institute) y su representante más destacado es Ole Wæver, que también fue el primero en teorizar en torno a la securitización. El COPRI funcionó como instituto independiente de investigación sufragado por el gobierno danés entre 1996 y 2003, año en que se fusionó con el Danish Institute for International Studies (DIIS).

${ }^{14}$ También referida como Escuela de Aberystwyth por la universidad en la que se desarrolló, es otra de las más influyentes y sus mayores representantes Ken Booth y Richard W. Jones.

15 MUTIMER, David, "Critical Security Studies: A schismatic history", en COLLINS, Alan (ed.) Contemporary Security Studies, Oxford University Press, Oxford, 2007, p. 59.

${ }^{16}$ La Escuela de París deriva de la tradición de Jacques Derrida y Michael Foucault, su más destacado representante es Didier Bigo, profesor de Sciences Po Paris y editor de la publicación trimestral en francés Cultures et Conflits, la cual ha sido asociada a esta Escuela. Entre sus grandes aportaciones está el estudio de las prácticas de seguridad y las actuaciones de las fuerzas de la autoridad, superando así la concepción original de la Escuela de Copenhague de la seguridad como acto del lenguaje. También podría incluirse en esta Escuela al experto belga de la Universidad de Namur, Thierry Balzacq quien ha enfatizado la importancia del contexto en que se producen los movimientos securitizadores y el papel que desempeñan para que la securitización se produzca (successful securitization) o falle (failed securitization).

${ }^{17}$ BUZAN, Barry, et. al., Security: a new framework..., op. cit., ps. 34-25. 
embargo teme las consecuencias de perpetuar, en un panorama político en rápido movimiento, las antiguas ortodoxias que propician el hambre, la enfermedad, la pobreza, la muerte y la opresión de millones de personas ${ }^{18}$. Además, Booth también distingue la teoría crítica de la seguridad del feminismo, de la Escuela de Copenhague, del constructivismo y del postmodernismo ${ }^{19}$.

\section{La evolución del estudio de la seguridad medioambiental}

En 1997, en un informe fruto de un proyecto de investigación conjunto del Peace Research Institute (PRIO) y el Fridtjof Nansen Institute (FNI), Rønnfeldt hacía una recapitulación de las tres generaciones por las que había pasado la investigación sobre seguridad medioambiental. Aunque sin grandes pretensiones, su segmentación en tres generaciones perseguía categorizar una serie de diferencias de base en los acercamientos académicos al campo de la seguridad medioambiental. A comienzos de la década de 1980, la primera generación estuvo dominada por el debate interdisciplinar tanto en la comunidad académica como en la política sobre si las cuestiones medioambientales debían tratarse como asuntos de seguridad y, de ser así, cómo deberían abordarse. Rønnfeldt señala que un punto de referencia es la crítica de Ullman a lo que este autor consideraba que era una interpretación demasiado estrecha de la seguridad nacional (defensa de las amenazas militares transfronterizas) y su propuesta de una definición más amplia del concepto.

La segunda generación surgió a principios de la década de 1990 en respuesta a las críticas vertidas por los tradicionalistas sobre la primera generación de que no aportaban evidencias empíricas ${ }^{20}$. Entre los exponentes destacados se encuentra Thomas Homer-Dixon, director del Proyecto sobre Medio Ambiente, Población y Seguridad de la Universidad de Toronto (de ahí la denominación "Grupo de Toronto"), cuyos numerosos estudios de casos tienen por objeto establecer un vínculo causal entre una variable independiente (escasez producida por cuestiones de índole medioambiental) y una variable dependiente (generación de conflictos violentos debido a la inestabilidad social). Quedaba así archivado el debate conceptual y se abría otro que, apoyándose sobre evidencias empíricas, exploraba la existencia y la naturaleza de los vínculos entre el medio ambiente y las amenazas clásicas de la seguridad.

Aunque el énfasis en los conflictos geopolíticos situaba a este grupo más cercano a la visión neorrealista de la seguridad como defensa, no terminó de convencer a un sector de la academia. Así, Barnett argumentaba que no se han hallado evidencias consistentes de que exista un vínculo causal entre la degradación medioambiental y el conflicto violento, señalando que las investigaciones se han centrado en una minoría de casos en que los conflictos han desembocado en violencia, pasando por alto que la mayoría de los problemas medioambientales se gestionan por medios no violentos.

A nuestro juicio, las críticas no restan valor a su gran aportación: los estudios empíricos que hicieron evolucionar la seguridad medioambiental por otras vías aportando evidencias de

\footnotetext{
${ }^{18}$ BOOTH, Ken (ed.), Critical Security Studies and World Politics, Lynne Rienner Publishers, Boulder, Colorado, 2005, p. 260.

19 MUTIMER, David, "Critical Security Studies..., op. cit., p. 64.

${ }^{20}$ RØNNFELDT, Carsten F., "Three generations of environment and security research", en Journal of Peace Research, no 34, 1997, ps. 474-75.
} 
la existencia de un vínculo entre seguridad y medio ambiente, aunque la escasez no sea la única causa de un conflicto violento sino más bien un factor coadyuvante cuando interactúa con otros factores de índole económica, política y social ${ }^{21}$.

La tercera generación, surgió a mediados de la década de 1990 y, por contraposición, los expertos buscaron ampliar el alcance de las variables dependientes para agregar a la investigación casos en que las cuestiones medioambientales condujeran a la cooperación. En esta etapa reina un cierto eclecticismo metodológico: estudios cuantitativos para confirmar las hipótesis del Grupo de Toronto, metodologías tomadas de las ciencias sociales como la teoría de los regímenes (Regime theory) que ofrece un marco analítico para comprender las condiciones bajo las cuales estados que se enfrentan a un problema común optan por establecer instituciones que les ayuden a resolverlo de forma cooperativa, o el enfoque del estado en la sociedad (state-in-society approach) que aborda la gobernanza en los países en desarrollo22.

Más recientemente, el profesor de la Universidad Libre de Berlín Hans Günther Brauch, ha actualizado y ampliado el trabajo de Rønnfeldt acotando tres fases en la investigación sobre medio ambiente y seguridad y, asimismo, delineando lo que a su juicio podría ser una cuarta fase de investigación en Seguridad Medioambiental y Humana y Paz (HESP, por sus siglas en inglés, que corresponden a Human and Environmental Security and Peace) ${ }^{23}$.

Las fases de Brauch coinciden a grandes rasgos con las generaciones de Rønnfeldt, si bien Brauch documenta más exhaustivamente los avances que se producen en cada etapa y, asimismo, amplía su alcance, lo cual en parte se debe a que mientras Rønnfeldt publica un artículo en una revista científica en un momento en que la seguridad medioambiental aún no tenía entidad propia, Brauch desarrolla su teoría en el marco de una obra cuasi enciclopédica diez años más tarde, en un momento en que la seguridad medioambiental ya ha entrado de lleno en la agenda política global con el cambio climático.

En la actualidad, el debate sobre si el medio ambiente se considera una cuestión de seguridad está superado con saldo positivo a favor de su inclusión, por lo que en los últimos años los expertos se han dedicado a desarrollar el campo ${ }^{24}$. No obstante, no existe una definición única de seguridad medioambiental, ni en el mundo académico de las Relaciones Internacionales, ni en la esfera política internacional.

Una de las primeras definiciones de seguridad medioambiental es la de Richard Ullman ${ }^{25}$ que en su artículo, Redefining Security, define qué es una amenaza a la seguridad

${ }^{21}$ Ibídem, p. 476.

22 Ibíd., p. 479.

${ }^{23}$ BRAUCH, Hans Günter, "Four phases of research on environment and security", en Encyclopedia of Life Support System, International Security, Peace, Development and Environment, 2007. También en BRAUCH, Hans Günter, et. al. (eds.), Facing global environmental change: Environmental, human, energy, food, health and water security concepts, Hexagon Series on Human and Environmental Security and Peace, Springer, 2009.

${ }^{24}$ FLOYD, Rita, Consequentialist evaluation of security for cooperative international society: a framework for analysis, ISA's 49th Annual Convention, Bridging Multiple Divides, San Francisco, 2008. http://research. allacademic.com/one/isa/isa08/index.php?click_key=1\#search_top [consultado el 19 de enero de 2015].

${ }^{25}$ ULLMAN, Richard H., "Redefining security", en International Security, no 8, 1983, p. 133. 
nacional en los siguientes términos:

"una acción o secuencia de sucesos que (1) amenaza drásticamente y en un periodo de tiempo relativamente breve con degradar la calidad de vida de los habitantes de un estado o, (2) amenaza significativamente con restringir el abanico de opciones políticas al alcance del gobierno de un estado o de entidades privadas no gubernamentales (personas, grupos, corporaciones) en el seno del estado."

Entre las amenazas medioambientales que podrían desestabilizar al estado, Ullman menciona la escasez de materias primas y desastres naturales devastadores, como epidemias virulentas, inundaciones catastróficas o sequías persistentes, pues podrían comprometer drásticamente el bienestar de una sociedad ${ }^{26}$. Aunque estas palabras nos pueden llevar a suponer que el bienestar de la sociedad es el objeto referente, lo que en realidad está amenazado es la estabilidad del Estado. No obstante, las palabras de Ullman dejan ya entrever que la perpetuación del Estado no es el único objeto referente que debe ser asegurado. Además, reconoce que las amenazas militares no son la única fuente de inestabilidad pues maneja un concepto amplio de seguridad con múltiples dimensiones (militar, política, económica, de la sociedad, medioambiental) y lo hace a varios niveles (sistema, estado, personas).

Tras sopesar varias definiciones de seguridad medioambiental en su libro The Meaning of Environmental Security, Barnett se decanta por un acercamiento de seguridad humana con la siguiente definición:

"El proceso de reducir por medios pacíficos la vulnerabilidad de los seres humanos a la degradación del medio ambiente causado por actividad humana, abordando las causas fundamentales de la degradación medioambiental y de la inseguridad humana"27.

El autor es consciente de que la seguridad medioambiental como condición absoluta es imposible. Sin embargo, al igual que la paz y la sostenibilidad, la seguridad medioambiental debería ser una meta sistemática, por lo que la define como un proceso adaptativo que es sensible al cambio y busca gestionarlo de forma pacífica ${ }^{28}$, es decir, a través de los mecanismos políticos ordinarios. Pero lo más llamativo es que Barnett define la seguridad medioambiental como un "proceso", pues a su juicio hasta el momento se había equiparado la seguridad con la inmovilidad.

Por otro lado, no todos los expertos que abogan por la seguridad medioambiental lo hacen del mismo modo, ni forman un bloque homogéneo. Existen diferentes interpretaciones o enfoques de lo que es y abarca la seguridad medioambiental, que vienen definidas en función a sus objetos referentes y los niveles a los que se tratan las fuentes de riesgo de cada una de ellas. Barnett analiza la literatura surgida desde comienzos de la década de 1990 en torno a la seguridad medioambiental y la clasifica en seis enfoques principales: seguridad

\footnotetext{
${ }^{26}$ Ibídem, p. 133.

27 BARNETT, Jon, The Meaning of Environmental Security: Ecological Politics and Policy in the New Security Era, Zed Books, Londres, 2001, p.129.

${ }^{28}$ Ibídem, p. 130.
} 
ecológica, seguridad común, violencia medioambiental, seguridad nacional, defensa verde, y seguridad $^{29}$.

\section{El marco de análisis de la Teoría de la Securitización de la Escuela de Copenhague}

La Teoría de la Securitización de la Escuela de Copenhague constituye uno de los enfoques críticos que mayor éxito ha tenido a la hora de abordar las cuestiones medioambientales en clave de seguridad, gracias en parte a la sencillez y efectividad de la aplicación de su concepto de securitización a una variedad de casos, en todos los sectores de la seguridad y en todos los continentes ${ }^{30}$. Sus postulados han sido inevitablemente comparados con los de la Escuela de Gales, que intencionalmente ha buscado diferenciarse de la primera proponiendo un marco de análisis orientado a la acción (ontología-epistemología-praxis).

Frente al carácter filosófico del debate sobre "qué" es seguridad que animó el intercambio de opiniones y definiciones en las publicaciones de estudios estratégicos y de seguridad, la Escuela de Copenhague introdujo un nuevo interrogante: ¿"Cómo" es la seguridad? Su obra clave Security: a new framework for analysis recoge el trabajo realizado en los años anteriores a su publicación y disperso en artículos académicos, sentando las bases de un marco analítico coherente fundamentado, por una parte, en el análisis sectorial y de niveles de Buzan y, por la otra, en el concepto de securitización de Wæver. El objetivo de dicho marco es poner en contexto cuestiones de seguridad que son presentadas como amenazas existenciales para un objeto referente por parte de un actor securitizador que, de esta manera, recaba apoyos para recurrir a medidas excepcionales que transgreden las normas que regirían de no ser crítica la situación. En resumen, son tres los elementos que intervienen en la securitización: un objeto referente, una amenaza a la supervivencia del mismo y un actor securitizador. A pesar de que en revisiones posteriores fue ganando importancia, originalmente el papel que desempeñaba el público o audiencia quedaba en segundo plano.

Además, se propone la utilización de niveles de análisis a fin de localizar actores, objetos referentes y dinámicas de interacción que operan en el terreno de la seguridad. Los cinco niveles de estudio más frecuentes en Relaciones internacionales son: sistemas internacionales, subsistemas internacionales (regionales o unidos por una preocupación común), unidades (estados, naciones, empresas transnacionales), subunidades (grupos de individuos organizados que operan dentro de las unidades, como las burocracias o los lobbies) y, por último, los individuos. Otro nivel de análisis son los sectores: el militar, el político, el económico, el social, y el medioambiental. Para que este acercamiento multisectorial tenga sentido, se toman en consideración nuevos objetos referentes y no solamente la seguridad del estado-nación.

\footnotetext{
${ }^{29}$ BARNETT, Jon, "Environmental Security", en COLLINS, Alan (ed.) Contemporary Security Studies, Oxford University Press, Oxford, 2007, p. 188.

30 Se habla también de la "Escuela de Singapur", encabezada por Mely Caballero-Anthony, que partiendo de los presupuestos de la Escuela de Copenhague y reconociendo que ofrece una perspectiva de constructivismo social para explicar cómo los problemas se transforman en asuntos de seguridad, desarrolla su propio marco de análisis para analizar cuestiones de seguridad no-tradicional en Non-traditional security in Asia: Issues, challenges and framework for action, un libro en el que se analizan varios casos utilizando una adaptación propia del marco de análisis de Security: a new framework for analysis.
} 
Pese a que han transcurrido casi 20 años desde que en 1998 se publicara, Security: a new framework for analysis ha superado con éxito la prueba del tiempo, pues en lo que respecta a la Teoría de la Securitización sigue siendo todavía hoy una obra de referencia citada en miles de artículos, cuyos planteamientos no solo son parte integrante de la Teoría de las Relaciones Internacionales y de los Estudios de Seguridad, sino que además han sido retomados por múltiples autores $y$, asimismo, revisados recientemente por varios críticos de la Teoría de Securitización tradicional, pertenecientes a la que Stritzel califica de "segunda generación crítica de la Teoría de la Securitización post-Escuela de Copenhague"131. De hecho, lejos de estar zanjado, el debate en torno al concepto de securitización continúa evolucionando en diferentes direcciones, y enriqueciéndose mediante su aplicación a múltiples casos de estudio, a varios niveles y en varios sectores.

El marco de la Teoría de la Securitización brinda una versátil herramienta para analizar riesgos o amenazas en todos los sectores, siendo el medioambiental uno de los más estudiados, tanto desde el punto de vista político como desde el social. Mutimer enfatiza la dimensión política de la seguridad al afirmar que la Securitización ha puesto sobre la mesa una serie de cuestiones de base que han dirigido el estudio crítico de la seguridad, preguntas como: "¿Quién puede securitizar un asunto de forma exitosa?", "¿Cuáles son las condiciones para que una securitización sea exitosa?" y "¿Cuáles son las consecuencias de la securitización?". Todas ellas confirman la naturaleza política de la seguridad y nos hacen plantearnos cómo los asuntos políticos y de seguridad se conforman de un determinado modo y cuáles son los procesos políticos que han llevado a dicha conformación ${ }^{32}$. Por su parte, Buzan y Hansen enfatizan su dimensión social cuando definen securitización como los "procesos sociales por los que un grupo de personas construyen algo en términos de amenaza"33.

En principio, cualquier cuestión pública podría situarse en cualquier punto de un recorrido que va de la no-politización a la politización y, de ahí, a la securitización. En otras palabras, independientemente de su importancia objetiva, cualquier asunto público podría no revertir ningún interés para el estado, por lo que no entraría en el debate político ni se tomarían decisiones al respecto (no-politización), o bien, requerir acción por parte del gobierno que debería pronunciarse al respecto y destinarle recursos (politización). Por último, existe la posibilidad de que un asunto se considere una amenaza existencial por lo que requeriría medidas y acciones excepcionales que rebasarían los límites de los procedimientos políticos (securitización) como podemos observar en el Gráfico 1. En este sentido, Buzan, et. al., señala que cuando un asunto rebasa los límites de la lógica política, incluso el propio proceso de establecer prioridades dejaría de tener sentido, pues "si no encontramos una solución a este problema, el resto de cosas resultará irrelevante (porque no estaremos aquí o no seremos libres para hacerles frente a nuestra manera) $)^{\prime 34}$.

\footnotetext{
${ }^{31}$ STRITZEL, Holger, Security in translation: Securitization theory and the localization of threat, Palgrave Macmillan, 2014.

32 MUTIMER, David, "Critical Security Studies..., op. cit., p. 61.

33 BUZAN, Barry y HANSEN, Lene, The evolution of..., op. cit., p. 37.

${ }^{34}$ BUZAN, Barry, et. al., Security: a new framework..., op. cit., p.24.
} 


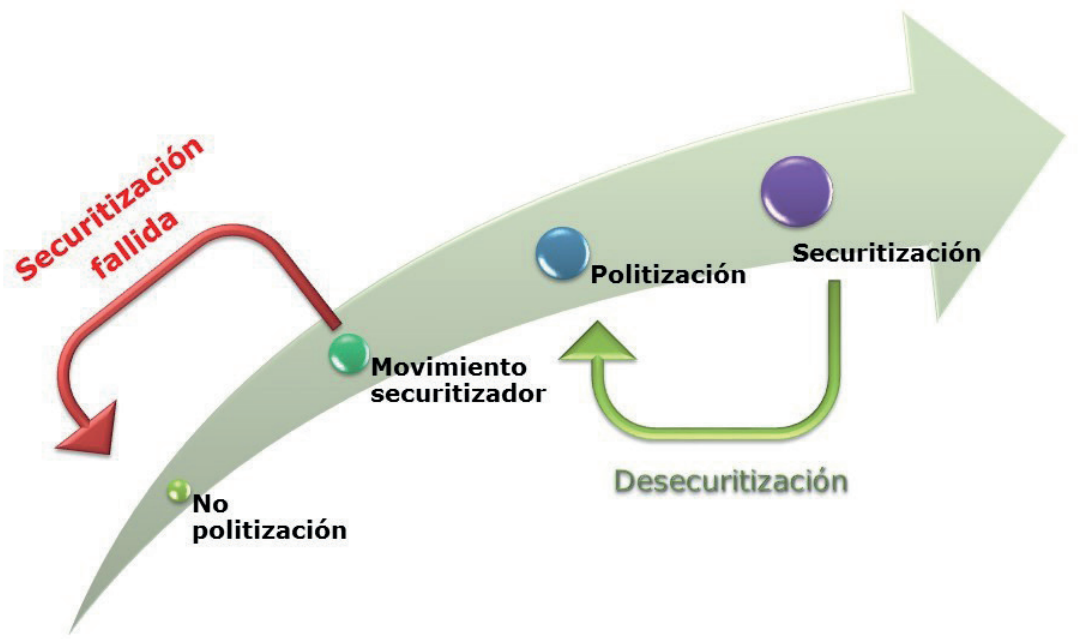

Fuente: Elaboración propia a partir de Buzan et al.

A juicio de Buzan et al., la seguridad es una práctica que es a la vez punto de partida y de llegada (self-referential), pues no es la existencia objetiva y real de un riesgo o una amenaza a la seguridad del estado -o a algún otro objeto de referencia- lo que la convierte en un asunto de seguridad, sino que es el hecho de calificar una cuestión como asunto de seguridad lo que la convierte en tal. Por otro lado, dicha declaración constituye un "movimiento securitizador" (securitizing move) que, sin embargo, no da lugar necesariamente a la securitización. Algunos movimientos securitizadores fracasan (failed securitization) y una securitización exitosa sólo se produce cuando un público acepta los argumentos que justifican que un asunto transgreda la agenda política ordinaria. Este carácter intersubjetivo es, por encima de la propia proclamación (self-referentiality), uno de los rasgos más destacables del proceso de securitización a juicio de varios autores que ven una contradicción en el hecho de que por una parte la Escuela de Copenhague alegue que la securitización es auto-referencial y, por la otra, que para ser legitimada sea necesario el apoyo de una masa crítica ${ }^{35}$.

La desecuritización es el proceso mediante el cual los asuntos que habían pasado a la pista rápida al ser securitizados, vuelven a la agenda política ordinaria donde otra vez son tratados de acuerdo a las normas establecidas. Para la Escuela de Copenhague, este movimiento significa la politización del asunto en cuestión y se considera algo positivo, pues lo contrario (la securitización) significa que no se ha logrado tratar el asunto siguiendo los cauces ordinarios.

${ }^{35}$ BALZACQ, Thiérry, "The three faces of securitization: Political agency, audience and context" en European Journal of International Relations, $\mathrm{n}^{\circ}$ 11(2), ps.171-201; STRITZEL, Holger, Security in translation... op. cit.; TROMBETTA, Maria Julia, "Rethinking the securitization... op. cit.; VERDES-MONTENEGRO ESCÁNEZ, Francisco J., "Securitización: agendas de investigación abiertas para el estudio de la seguridad" en Relaciones Internacionales, no 29, 2015, ps. 111-131. 
En opinión de Wæver ${ }^{36}$, la seguridad es lo contrario de la política. Seguridad y securitización constituyen un fracaso de las vías políticas ordinarias, en las que existe más libertad para entrar en debate y entablar un diálogo abierto. En clave de seguridad, por el contrario, prima la inmediatez y la búsqueda de remedios a corto plazo. "La desecuritización es la opción óptima a largo plazo"37. Su visión de la seguridad como un fracaso de la política convencional continúa presente en trabajos posteriores, en los que Weaver esgrime una preferencia por la desecuritización, argumentando que la securitización es un desarrollo negativo, implícito en la dicotomía seguridad y política.

\section{El cambio climático como ejemplo de securitización exitosa del medio ambiente}

La securitización del medio ambiente ha logrado poner de relieve determinados riesgos medioambientales que habían quedado relegados al segundo plano por asuntos más inmediatos como la amenaza terrorista. Así, por ejemplo, a finales de década de 1980 el reconocimiento colectivo de riesgos globales como el transporte aéreo de partículas contaminantes a larga distancia, la reducción de la capa de ozono, el calentamiento global o la sobreexplotación de los caladeros mundiales, dio paso a negociaciones internacionales ${ }^{38}$ que desembocaron en la firma de convenciones vinculantes. Fue el tratamiento en clave de seguridad lo que hizo que se les dedicaran esfuerzos extraordinarios que por la vía política ordinaria habrían tardado más en llegar o no lo habrían hecho.

Revisamos a continuación, siguiendo el marco de análisis de la Escuela de Copenhague, cuáles son las amenazas, los objetos referentes y los distintos actores que toman parte en los procesos de securitización medioambiental. Asimismo, estudiamos el proceso de securitización que ha experimentado el cambio climático, pasando de ser considerado una cuestión ecológica a convertirse en un problema de seguridad medioambiental global que ha dado lugar a la creación de un régimen para la reducción de gases de efecto invernadero (en adelante, GEI).

\subsection{Amenazas medioambientales}

La naturaleza global a largo plazo la mayor parte de las amenazas medioambientales no encaja con el enfoque tradicional de la seguridad nacional, centrado en amenazas militares e inmediatas. La seguridad medioambiental requiere de un enfoque crítico dada la amplitud de las fuentes que pueden provocar inseguridad, desde los desastres naturales a la degradación del medio ambiente provocada por la acción humana, pasando por los denominados "natechs" desastres naturales agravados por la acción humana cada vez más frecuentes en nuestra "sociedad del riesgo" (risk society) como pone de manifiesto el último gran accidente nuclear, el de Fukushima en Japón, que alcanzó un nivel 7 en la escala INES, lo cual lo sitúa a la par con Chernóbil.

Aunque existen varias clasificaciones de las tipologías de riesgos medioambientales, a grandes rasgos podríamos establecer tres tipos atendiendo a la intervención del ser humano

\footnotetext{
${ }^{36}$ W/EVER, Ole, Concepts of security (PhD thesis), University of Copenhagen, Institute of Political Science, 1997.

37 BUZAN, Barry, et. al., Security: A new framework..., op. cit., p.29.

${ }^{38}$ LIDSKOG, Rolf; SUNDQVIST, Göran. "The role of science in environmental regimes: The case of LRTAP" en European Journal of International Relations, vol. 8, nº 1, 2002, p.79.
} 
en su generación. En primer lugar, los riesgos medioambientales no causados por los seres humanos, como huracanes y otros desastres naturales. En segundo lugar, las amenazas medioambientales generadas por la actividad humana que amenazan a la humanidad, por ejemplo, las emisiones de GEI. En tercer lugar, las amenazas medioambientales creadas por la actividad humana que no amenazan directamente al ser humano, como el agotamiento de los recursos minerales o la extinción de determinadas especies de flora y fauna.

Los riesgos del primer tipo son explícitamente securitizados y están institucionalizados. Por ejemplo, en los países donde se dan huracanes, la declaración del estado de emergencia cuando se aproxima uno se lleva a cabo de oficio. Un ejemplo para el segundo tipo es la declaración de estado de emergencia nuclear en caso de producirse un accidente en una central nuclear, que permite tomar medidas excepcionales sin tener que seguir los procesos políticos de debate habituales en la vida democrática, pudiéndose tomar medidas como la intervención de las fuerzas armadas en las labores de socorro o la evacuación de zonas de riesgo, sin tener en cuenta las libertades individuales. De hecho, la simple provisión en la legislación de un estado de emergencia, es en sí una medida excepcional.

Si bien los del tercer tipo constituyen el riesgo medioambiental más genuino, son los del segundo tipo los que se abordan con más frecuencia, tanto en la agenda científica como en la política, dado que son cuestiones en las que la intervención humana puede cambiar el curso de los acontecimientos. Este es, por ejemplo, el caso del cambio climático. Según el Grupo Intergubernamental para el Cambio Climático (en adelante, IPCC por sus siglas en inglés que corresponden a International Panel on Climate Change), desde 1900 la atmósfera se ha calentado casi $1^{\circ} \mathrm{C}$, haciendo que las actuales temperaturas sean las más elevadas en el actual periodo interglaciar que comenzó hace 12.000 años. Las emisiones de GEI están produciendo una alteración climática que será irreversible si se supera el límite teórico de los $2^{\circ} \mathrm{C}$ sobre la temperatura existente en los tiempos preindustriales.

A medida que el estudio científico ha ido logrando una mejor comprensión del fenómeno, ha pasado de considerarse un problema medioambiental a conceptualizarse como un reto para la sostenibilidad global por su incidencia en los ámbitos sociales y económicos. De forma que, aunque el IPCC no entra a hacer juicios, sino que expone sus hallazgos de forma objetiva y además utilizando un lenguaje bien matizado para definir el grado de certeza de sus conclusiones ${ }^{39}$, distintos actores securitizadores con distintos puntos de vista e intereses, definen distintos objetos referentes y amenazas, defendiendo asimismo distintos argumentos, como veremos a continuación.

\subsection{Actores: ¿Quiénes tienen capacidad de securitizar un tema medioambiental?}

Buzan, et. al., identifican tres tipos de actores: los actores principales, actores de veto y actores de apoyo (lead, veto and support actors, respectivamente). Los actores principales poseen un firme compromiso de acción respecto a una determinada cuestión medioambiental, como actores securitizadores tienen capacidad de recabar apoyos de un público (audience) y poner en funcionamiento medidas extraordinarias.

${ }^{39}$ IPCC, Guidance note for lead authors of the IPCC Fifth Assessment Report on consistent treatment of uncertainties. [https://www.ipcc.ch/pdf/supporting-material/uncertainty-guidance-note.pdf] 
¿Quiénes pueden desempeñar este papel? En la agenda científica son los científicos, mientras que en la política algunos actores están mejor posicionados que otros para securitizar un asunto. Aunque en el marco original de la Escuela de Copenhague este papel parecía estar reservado a dirigentes y políticos de alto nivel, la práctica demuestra que también poseen capacidad de securitizar otros actores como lobbies y ONGs cuyo campo de acción es el medio ambiente (por ejemplo, Greenpeace, Friends of the Earth o WWF). Estos grupos de presión pueden crear conciencia sobre un problema mediante la financiación de investigaciones y campañas informativas dirigidas a la opinión pública, organizar acciones de forma unilateral o recurrir a la diplomacia para introducir un tema en la agenda de organizaciones internacionales con mayor capacidad securitizadora.

Por su parte, los actores de veto también pueden ser ONGs, empresas transnacionales y lobbies que restan importancia a las cuestiones medioambientales cuando ven cuestionados sus intereses. Así, durante años los negacionistas del cambio climático, anteponiendo los intereses económicos de unos pocos a la protección de los bienes comunes globales ( $\mathrm{global}$ commons), argumentaban que proteger el medio ambiente constituía un obstáculo para el desarrollo, entre otras cosas. En lo que respecta al cambio climático, dado que las sólidas evidencias presentadas por la comunidad científica hablan por sí solas, cada vez son más escasos quienes defienden la posición contraria. Por último, los actores de apoyo carecen de recursos para liderar una causa por lo que se limitan a apoyar las causas presentadas por los actores principales. En torno a cada cuestión se moviliza una constelación de actores propia, característica de cada coyuntura.

El tratamiento del cambio climático a través del IPCC con sus Informes de Evaluación es un buen ejemplo de cómo el conocimiento experto de la comunidad científica ha influido sobre las decisiones políticas de la comunidad internacional, y cómo esta ha ido movilizando recursos para crear un régimen de reducción de emisiones con compromisos vinculantes para las partes firmantes. Cada actor securitizador interpreta el cambio climático desde su propia perspectiva, así para las ONGs y OIGs, para los ecologistas e incluso para la comunidad científica el principal causante son las emisiones de GEI propiciadas por la forma de vida de las sociedades industrializadas y las prácticas de algunos gobiernos sin un firme compromiso con los esfuerzos de detener el cambio climático, ya que la urgencia de actuar no es la misma para gobiernos que lo consideran algo remoto y alejado de sus preocupaciones inmediatas, que además conlleva un coste económico, que para las naciones-isla del Pacífico Sur que corren el riesgo de desaparecer si continúa subiendo el nivel del mar.

Los estados, por su parte, ven en el cambio climático un "multiplicador de las amenazas" dada su capacidad tanto de agravar conflictos preexistentes, como de exacerbar fenómenos climáticos extremos (huracanes, tifones y otros desastres naturales), lo cual les podría desestabilizar. Por esta razón, algunos gobiernos tratan el tema en su estrategia nacional de seguridad. En la misma línea, para organizaciones internacionales como el Foro Económico Mundial (WEF) el cambio climático constituye una amenaza para la estabilidad internacional, por sus efectos negativos sobre la economía, aunque en este caso el objetivo es encontrar apoyos y fomentar la colaboración internacional. 


\subsection{Objetos referentes}

Tomando como objeto referente el estado, el concepto de seguridad medioambiental se ligó a las guerras causadas por la escasez de recursos (resource wars), una teoría apoyada por autores como Ullman o Homer-Dixon; mientras otros autores establecen como objeto referente el medio ambiente en sí mismo, amenazado por factores como el cambio climático o la degradación de la tierra, la biodiversidad, la atmósfera, el agua, los bosques, las costas y los ríos $^{40}$. Esta línea está en consonancia con las teorías de la precursora del medioambientalismo Rachel Carson, que con su influyente obra de 1962 "La Primavera Silenciosa" creaba conciencia de que la acumulación de pesticidas en la cadena alimentaria producida por los vertidos industriales estaba poniendo en riesgo el medioambiente de las zonas rurales de Estados Unidos, una situación que podría repetirse a nivel internacional.

Por su parte, el Programa de las Naciones Unidas para el Desarrollo desplazó el foco a las personas al preocuparse por "cómo viven y respiran en una sociedad, con qué libertad pueden ejercer sus decisiones, qué acceso tienen a las oportunidades sociales y de mercado $y$, si viven en conflicto o en paz"41.

En el cambio climático confluyen todos estos objetos referentes. Resulta problemático aislar unos efectos de otros e igualmente sucede con los objetos referentes, ya que la degradación del medio ambiente y el cambio climático ponen en riesgo al propio medio ambiente (seguridad ecológica), a las personas y su forma de vida (seguridad humana), a los estados (seguridad nacional) y la estabilidad del orden internacional (seguridad internacional). La securitización del cambio climático ha conseguido recabar apoyos por parte de la comunidad internacional, si bien desde la perspectiva de la seguridad nacional los países menos desarrollados son más vulnerables al cambio climático, mientras que desde la perspectiva de la seguridad humana los más vulnerables son las mujeres, los niños, los ancianos y los pobres.

En la Tabla 1 hacemos una recapitulación lo anteriormente expuesto, consignando amenazas, actores y objetos referentes que entran en juego dentro del cambio climático, cada uno siguiendo una línea argumental diferenciada.

${ }^{40}$ BARNETT, Jon, "Environmental Security", op. cit., 189.

41 UNITED NATIONS DEVELOPMENT PROGRAMME, Human Development Report 1994, Oxford University Press for the United Nations Development Programme (UNDP), Nueva York, 1994, p.23. 
Tabla 1. Cuadro resumen enfoques cambio climático

\begin{tabular}{|c|c|c|c|c|}
\hline $\begin{array}{l}\text { Actor } \\
\text { securitizador }\end{array}$ & Amenaza & Objeto referente & Enfoque & Antecedentes \\
\hline $\begin{array}{l}\text { ONGs } \\
\text { ambientalistas } \\
\text { Comunidad } \\
\text { científica }\end{array}$ & $\begin{array}{c}\text { Forma de vida de las } \\
\text { sociedades industriales } \\
\text { y prácticas de algunos } \\
\text { gobiernos }\end{array}$ & Medio ambiente & Ecologismo & Rachel Carson \\
\hline OIGs, ONGs & $\begin{array}{c}\text { Forma de vida de las } \\
\text { sociedades industriales } \\
\text { y prácticas de algunos } \\
\text { gobiernos }\end{array}$ & $\begin{array}{l}\text { Ser humano. Forma de } \\
\text { vida de las personas }\end{array}$ & $\begin{array}{l}\text { Seguridad } \\
\text { humana }\end{array}$ & UNEP \\
\hline Estado & $\begin{array}{c}\text { Efecto multiplicador del CC. } \\
\text { Agravante de conflictos pre- } \\
\text { existentes }\end{array}$ & Estado & $\begin{array}{l}\text { Seguridad } \\
\text { nacional }\end{array}$ & Grupo de Toronto \\
\hline $\begin{array}{l}\text { Organizaciones } \\
\text { internacionales }\end{array}$ & Poder desestabilizador del CC & $\begin{array}{l}\text { Estabilidad del orden } \\
\text { internacional }\end{array}$ & $\begin{array}{c}\text { Seguridad } \\
\text { internacional }\end{array}$ & UNEP \\
\hline
\end{tabular}

Fuente: elaboración propia

\subsection{La doble agenda de la seguridad medioambiental y las comunidades epistémicas}

El sector medioambiental se caracteriza por promover dos agendas, una científica y otra política, que se configuran por influencia mutua, además de solaparse en los medios de comunicación y en los debates sociales ${ }^{42}$. La agenda política está dominada por gobiernos y organizaciones intergubernamentales, en ella se lleva a cabo el proceso político de toma de decisiones y se elaboran políticas públicas en materia de medio ambiente, en función de las cuales se asignan recursos. La agenda científica, por su parte, se apoya sobre la evaluación de las evidencias científicas por parte de expertos que con su juicio contribuyen a que una cuestión medioambiental sea securitizada o desecuritizada.

A pesar de su solapamiento e interdependencia, ambas agendas difieren en que la agenda política se ajusta a estándares públicos, gubernamentales y periodísticos, que se mueven más en el corto plazo, mientras que en la agenda científica predomina el rigor de cumplir con estándares académicos. Así, para la agenda política resulta crítico determinar si la gravedad de un asunto constituye o no una cuestión política.

Las cuestiones medioambientales son analizadas en primera instancia por científicos y académicos que los incluyen en la agenda científica, desde la que los temas más destacados ascienden a la agenda política. Un problema medioambiental podría pasar varios años en la agenda científica antes de ser politizado o priorizado (securitizado), logrando ascender a la agenda política. Ese fue el caso, por ejemplo, de la lluvia ácida en Europa. Aunque los primeros daños provocados por la acidez del agua pluvial se manifestaron en los bosques escandinavos en la década de 1950, sus efectos no se estudiaron en profundidad ni se consiguió probar que existía una correlación entre las emisiones de azufre de las fábricas rusas y la acidificación de lagos y bosques escandinavos hasta finales de la década de 1960. La lluvia ácida no

${ }^{42}$ BUZAN, Barry, et. al., Security: a new framework..., op. cit., p.71. 
entró en la agenda política hasta la década de 1970 y, transcurridos casi 30 años desde los primeros indicios, finalmente las negociaciones intergubernamentales concluyeron en 1979 con la firma Convención sobre el Transporte a Larga Distancia de Contaminantes Atmosféricos Transfronterizos (CLRTAP, por sus siglas en inglés que corresponden a Convention on Longrange Transboundary Air Pollution) ${ }^{43}$.

En todo este proceso desempeñaron un papel determinante las comunidades epistémicas, cuyo objetivo primordial es construir conocimiento orientado a la formulación de políticas. Hass las define como una red transnacional de profesionales basada en el conocimiento cuyo poder político reside en su autoridad cognitiva, procedentes de diferentes disciplinas pero con un corpus de conocimientos y valores compartidos ${ }^{44}$.

Además de compartir hipótesis sobre las relaciones causa-efecto y sus posibles soluciones, el conocimiento consensuado que generan es capaz de influir sobre las actitudes de los actores que forman parte de un régimen. A juicio de Lidskog y Sundqvist, la LTRAP ilustra bien este punto, pues la interacción entre los integrantes de la comunidad epistémica impulsó otras formas de percibir el problema de la lluvia ácida, sus efectos y como abordarlo de forma exitosa ${ }^{45}$. Los autores enfatizan la relevancia del conocimiento consensuado, ya que "el conocimiento científico y el orden político se conforman mutuamente a través de un proceso interdependiente de evolución"46, es decir, existe una "coproducción de ciencia y política" de manera que el valor de la ciencia es producto de un proceso de negociación.

Establecido en 1988 por recomendación de UNEP (Programa del Medio Ambiente de la ONU), el IPCC es otro ejemplo clásico de comunidad epistémica. Se trata de un foro abarcador donde se presentan las evidencias de científicos y expertos en forma de Informes de Evaluación ("Assessment Reports" o ARs), en cuya elaboración intervienen más de un millar de científicos independientes, que analizan los últimos estudios científicos sobre cambio climático aportados por expertos de todo el mundo, ya que no llevan a cabo investigaciones propias. Desde el punto de vista organizativo, este órgano científico e intergubernamental en el que participan 195 países, cuenta con una presidencia y un secretariado con sede en Ginebra. Su trabajo se coordina a través de una serie de grupos de trabajo, cuyos hallazgos proporcionan a los gobiernos una base científica para la toma de decisiones. Hasta el momento han visto la luz cinco ARs (1990, 1995, 2001, 2007 y el último en 2014) y actualmente están trabajando en un sexto, cada uno de ellos actualiza los datos del anterior porque cada vez hay más estudios y más avances tecnológicos que proporcionan datos más fiables. En la Tabla 2, podemos ver como su historia corre paralela al avance en el establecimiento de un régimen internacional que persigue mitigar el cambio climático.

Desde mediados de la década de 1970, los analistas de seguridad partidarios de la "ampliación" del concepto de seguridad comenzaron a hacerse eco de las implicaciones

\footnotetext{
43 Para ampliar información y acceder al texto de la Convención: http://www.unece.org/env/Irtap/Irtap_h1.html

${ }^{44}$ Citado en LIDSKOG, Rolf; SUNDQVIST, Göran. "The role of science... op. cit., p.82.

45 Ibídem, p.83.

${ }^{46}$ Ibíd., p.84.
} 
de varias amenazas ambientales entre las que se contaba el cambio climático ${ }^{47}$, así en su aclamado informe de 1977 del Worldwatch Institute, Lester Brown trataba "the threat of climate modification". Sin embargo, un momento clave para la securitización del cambio climático fue la reunión del G7 en Toronto en 1988, en que la administración Reagan lo puso en la agenda, junto a otras cuestiones de seguridad tradicional como el terrorismo, la economía global o las relaciones con la Unión Soviética ${ }^{48}$. Este movimiento securitizador encontró un público receptivo, dando lugar a una securitización exitosa del cambio climático que de esta manera entró en la agenda internacional y posteriormente se materializó con la creación del IPCC, en el plano científico, y en el plano político, con la firma de la Convención Marco sobre Cambio Climático de la ONU (UNFCCC), que entró en vigor en 1994, así como con las sucesivas reuniones de las partes firmantes de dicha convención, las denominadas COP.

El último hito en la historia de los esfuerzos por mitigar el cambio climático es el Acuerdo de París que salió de la COP21 en diciembre 2015 y tiene por objeto contener el calentamiento global por debajo de $2^{\circ} \mathrm{C}$ con respecto a los niveles pre-industriales. Frente al principio de "responsabilidad común pero diferenciada" (CBDR) que había estado presente en arreglos anteriores y disponía que los países desarrollados ejerciesen el liderazgo, tanto en materia de reducción de emisiones como en la provisión de medios de cara a los países en desarrollo ${ }^{49}$, el Acuerdo de París marca un hito histórico en la lucha contra el calentamiento global, por ser el primer marco que exige a todos los países, tanto desarrollados como en desarrollo, que adopten medidas concretas para reducir sus emisiones de GEI bajo el modelo de "Contribuciones Previstas Determinadas a Nivel Nacional" (INDC, Intended Nationally Determined Contributions), según el cual cada estado determina su contribución de acuerdo a sus prioridades nacionales, circunstancias y capacidades.

Para no detener el proceso, se estableció que el Acuerdo de París entraría en vigor una vez fuera ratificado por 55 países que representasen al menos el 55\% de las emisiones globales de GEI. En septiembre de 2016 ya lo habían ratificado más de 60 países, entre los que se contaban los dos que más GEI emiten (Estados Unidos y China), sin embargo apenas suponían el $48 \%$ de las emisiones. No obstante, tras una nueva ronda de ratificaciones durante la celebración de la Asamblea General de la ONU de septiembre de 2016, el Secretario General de la ONU, Ban Ki-moon, se mostraba convencido que el Acuerdo de París podría entrar en vigor antes de finalizar el año, asegurando que la lucha contra el cambio climático había constituido una prioridad durante sus diez años de mandato. El 5 de octubre de 2016 se alcanzaba el umbral establecido, por lo que el 4 de noviembre de 2016 entraba en vigor.

La COP22, celebrada en Marrakech del 7 al 18 de noviembre de 2016, confirmó el compromiso internacional de continuar por la senda marcada, independientemente del rumbo que tomase Estados Unidos tras la elección como presidente de Donald Trump en unas elecciones que coincidieron en el tiempo con la cumbre ( 8 de noviembre). De hecho, se

\footnotetext{
${ }^{47}$ BROWN, Lester Russell, Redefining national security, vol. 14, Worldwatch Institute, Washington D.C. 1977, ps.20-24.

${ }^{48}$ HOUGH, Peter, Environmental security: an introduction, Routledge, 2014, p.70.

${ }^{49}$ BUENO RUBIAL, María del Pilar, "El Acuerdo de París: ¿una nueva idea sobre la arquitectura climática internacional?", Relaciones Internacionales, no 33, 2016, p. 80.
} 
avanzó en la redacción de normas de aplicación del Acuerdo de París y anunciaron nuevos compromisos no solo los gobiernos nacionales, sino también empresas, inversores, ciudades y gobiernos locales. Pero lo más destacado es que los negociadores acordaron aplicar los compromisos del Acuerdo de Paris a corto plazo, hasta 2018, año en que se celebrará otra cumbre climática en la que las partes deberán presentar sus planes de reducción mejorados tras 2020, mientras que la COP23 tendrá lugar en Bonn en $2017^{50}$.

Tabla 2. Hitos en la carrera del ipcc

Fuente: IPCC

\begin{tabular}{|c|c|}
\hline 1979 & Conferencias sobre el clima mundial \\
\hline 1988 & Se establece el IPCC \\
\hline 1990 & $\begin{array}{l}\text { Primer Informe de Evaluación (AR1) del IPCC. } \\
\text { La ONU pone en marcha negociaciones de una convención marco sobre cambio climáticc }\end{array}$ \\
\hline 1991 & Primera reunión del IPCC (Grupo Intergubernamental para el Cambio Climático) \\
\hline 1992 & $\begin{array}{l}\text { Cumbre de la Tierra (Rio de Janeiro). } \\
\text { Se abre a la firma la UNFCCC (Convención Marco sobre Cambio Climático) }\end{array}$ \\
\hline 1994 & La UNFCCC entra en vigor \\
\hline 1995 & $\begin{array}{l}\text { Primera Conferencia de las Partes (COP1) en Berlín. } \\
\text { Segundo Informe de Evaluación (AR2) }\end{array}$ \\
\hline 1996 & Comienza operaciones el Secretariado de UNFCCC \\
\hline 1997 & Se adopta el Protocolo de Kioto (COP3) \\
\hline 2001 & Tercer Informe de Evaluación (AR3) \\
\hline 2005 & El Protocolo de Kioto entra en vigor \\
\hline 2007 & El IPCC publica su Cuarto Informe de Evaluación (AR4) \\
\hline 2009 & Acuerdo de Copenhague (COP15) \\
\hline 2010 & Acuerdos de Cancún (COP16) \\
\hline 2014 & Quinto Informe de Evaluación (AR5) \\
\hline 2015 & Cumbre de París (COP 21) \\
\hline 2016 & $\begin{array}{l}\text { Entra en vigor el Acuerdo de Paris } \\
\text { Cumbre de Marrakech (COP 22) }\end{array}$ \\
\hline
\end{tabular}

\section{Conclusión}

La gestión que la comunidad internacional está haciendo del cambio climático constituye un excelente paradigma de securitización exitosa del medio ambiente que pone de manifiesto que determinadas cuestiones de seguridad medioambiental pueden suponer un riesgo existencial, por lo que es necesario dedicarles medios extraordinarios (creación del IPCC, establecimiento del UNFCCC, seguimiento a través de las CoPs) y esfuerzos también extraordinarios (colaboración internacional, firma de acuerdos vinculantes).

La interrelación mutuamente influyente de científicos y dirigentes políticos es una

\footnotetext{
50 Para ampliar información, véase el comunicado de prensa emitido al finalizar el encuentro http://newsroom. unfccc.int/es/noticias/cop22-comunicado-de-prensa-final/
} 
de las razones del éxito. Podemos concluir, por tanto, que las comunidades epistémicas desempeñan un papel protagonista en el tratamiento de temas medioambientales en clave de seguridad, logrando que se pongan en marcha mecanismos extraordinarios que aceleren los procesos y muevan a la acción, aunque sin salirse de los cauces ordinarios de la política, donde imperan el debate y la negociación. No obstante, gracias a su tratamiento en modo de emergencia, las negociaciones se están cerrando en un tiempo inferior al que suelen llevar otras negociaciones internacionales y los compromisos alcanzados son cada vez más ambiciosos.

En este sentido, el Acuerdo de París fruto de la COP21 constituye un hito al conseguir poner de acuerdo a todas las partes, más aún tras el decepcionante resultado del COP15 del que salió un acuerdo de mínimos (Acuerdo de Copenhague). No obstante, queda por comprobar si desde una doble perspectiva los acuerdos e instrumentos actuales resultan eficaces para frenar el cambio climático: por una parte, que las acciones propuestas en materia de mitigación y adaptación consigan frenar el calentamiento global. Por la otra, que los países firmantes cumplan con lo prometido voluntariamente, especialmente teniendo en cuenta que el objetivo final sería alcanzar una economía de emisiones cero en la segunda mitad del siglo XXI y que aplicar sanciones para hacerlos cumplir plantea serias dificultades.

\section{Bibliografía}

ALLENBY, Braden R., "Environmental security: Concept and implementation", en International Political Science Review, no 21, 2000, ps. 5-21.

BALZACQ, Thiérry, "The three faces of securitization: Political agency, audience and context" en European Journal of International Relations, n ${ }^{011}(2), \mathrm{ps} .171-201$.

BARNETT, Jon, "Environmental Security", en COLLINS, Alan (ed.) Contemporary Security Studies, Oxford University Press, Oxford, 2007, ps. 182-203.

BARNETT, Jon, The Meaning of Environmental Security: Ecological Politics and Policy in the New Security Era, Zed Books, Londres, 2001.

BOOTH, Ken, "Security and emancipation", en Review of International Studies, no 17 (04), 1991, ps. 313-326.

BOOTH, Ken (ed.), Critical Security Studies and World Politics, Lynne Rienner Publishers, Boulder, Colorado, 2005.

BRAUCH, Hans Günter, "Four phases of research on environment and security", en Encyclopedia of Life Support System, International Security, Peace, Development and Environment, 2007.

BRAUCH, Hans Günter, et.al. (eds.), Facing global environmental change: Environmental, human, energy, food, health and water security concepts, Hexagon Series on Human and Environmental Security and Peace, Springer, 2009.

BROWN, Lester Russell, Redefining national security, vol. 14, Worldwatch Institute, Washington D.C., 1977.

BUENO RUBIAL, María del Pilar, "El Acuerdo de París: ¿una nueva idea sobre la arquitectura climática internacional?", Relaciones Internacionales, no 33, 2016, ps.75-95.

BUZAN, Barry, WÆVER, Ole y DE WILDE, Jaap, Security: a new framework for analysis, Lynne Rienner Publishers, Londres, 1998.

BUZAN, Barry y HANSEN, Lene, The evolution of International Security Studies, Cambridge University Press, 2009.

DEUDNEY, Daniel H., "Environmental security: A critique" en DEUDNEY, Daniel H. y MATTHEW, Richard 
A. (eds.), Contested Grounds: Security and Conflict in the New Environmental Politics, SUNY Press, Albany, Nueva York, 1999, ps. 187-219.

DEUDNEY, Daniel, "Environment and Security: Muddled Thinking", en Bulletin of the Atomic Scientists, no 47, 1991, ps. 22-28.

FLOYD, Rita, Consequentialist evaluation of security for cooperative international society: a framework for analysis, ISA's 49th Annual Convention, Bridging Multiple Divides, San Francisco, 2008. http:// research.allacademic.com/one/isa/isa08/index.php?click_key=1\#search_top [consultado el 19 de enero de 2015].

HOMER-DIXON, Thomas F., "On the Threshold: Environmental Changes as Causes of Acute Conflict", en International Security, 16 (2), 1991, ps. 76-116, doi: 10.2307/2539061.

HOMER-DIXON, Thomas F., "Thresholds of Turmoil: Environmental Scarcities and Violent Conflict", DEUDNEY, Daniel H., y MATTHEW, Richard A. (eds.), Contested grounds: security and conflict in the new environmental politics, SUNY Press, Albany, Nueva York, 1999, ps. 61-90.

HOUGH, Peter, Environmental Security: An introduction, Routledge, Londres, 2014.

KRAUSE, Keith, y WILLIAMS, Michael C., "Broadening the agenda of security studies: politics and methods", en Mershon International Studies Review, no 40, 1996, ps. 229-54, doi: 10.2307/222776.

LIDSKOG, Rolf; SUNDQVIST, Göran, "The role of science in environmental regimes: The case of LRTAP" en European Journal of International Relations, vol. 8, no 1, 2002, ps. 77-101.

MUTIMER, David, "Critical Security Studies: A schismatic history", en COLLINS, Alan (ed.) Contemporary Security Studies, Oxford University Press, Oxford, 2007, ps. 53-74.

RøNNFELDT, Carsten F., "Three generations of environment and security research", en Journal of Peace Research, no 34, 1997, ps. 473-82.

STRITZEL, Holger, Security in translation: Securitization theory and the localization of threat, Palgrave Macmillan, 2014.

TROMBETTA, Maria Julia, "Rethinking the securitization of the environment: Old beliefs, new insights", en BALZACQ, Thierry (ed.), Securitization Theory: How security problems emerge and dissolve, Routledge, Londres, 2011, ps. 135-49.

ULLMAN, Richard H., "Redefining security", en International Security, no 8, 1983, ps. 129-53, doi: $10.2307 / 2538489$.

UNITED NATIONS DEVELOPMENT PROGRAMME, Human Development Report 1994, Oxford University Press for the United Nations Development Programme (UNDP), Nueva York, 1994.

VERDES-MONTENEGRO ESCÁNEZ, Francisco J., "Securitización: agendas de investigación abiertas para el estudio de la seguridad", Relaciones Internacionales, no 29, 2015, ps. 111-131.

WÆVER, Ole, Concepts of security (PhD thesis), University of Copenhagen, Institute of Political Science, 1997. 


\section{RELACIONES INTERNACIONALES}

Revista académica cuatrimestral de publicación electrónica Grupo de Estudios de Relaciones Internacionales (GERI) Universidad Autónoma de Madrid, España

www.relacionesinternacionales.info

ISSN 1699 - 3950

ff facebook.com/RelacionesInternacionales

twitter.com/RRInternacional 